\title{
Senemorphism: a novel perspective on aging patterns and its implication for diet-related biology
}

\author{
Lucas Siqueira Trindade • Alex Balduino • \\ Toshiro Aigaki · Jonathan G. Heddle
}

Received: 25 January 2012/Accepted: 20 April 2012/Published online: 4 May 2012

(C) The Author(s) 2012. This article is published with open access at Springerlink.com

\begin{abstract}
Aging can be described as the accumulation of changes in organisms over time. Aging in organisms undergoing caloric restriction (CR) is widely considered as a slowed version of aging under ad libitum (AL) conditions. However, here we argue that aging under optimized CR is fundamentally different from aging under AL based on the following facts: (1) Comparing the two dietary groups, several age-related changes run in the opposite direction over time; (2) Switching from an AL to a CR diet clearly reverts (not only delays) several "normal" accumulated changes; (3) major causes of death are as different between both groups as they are between species. These observations support the idea that CR
\end{abstract}

\section{S. Trindade $(\bowtie) \cdot$ J. G. Heddle}

Heddle Initiative Research Unit, Advanced Science Institute, 2-1 Hirosawa, Wako, Saitama 351-0198, Japan e-mail: lucas.t@riken.jp

\section{S. Trindade}

Department of Investigative Pathology, Graduate School of Biomedical Sciences, Nagasaki University,

Nagasaki 852-8523, Japan

L. S. Trindade · T. Aigaki

Department of Biological Sciences, Tokyo Metropolitan University, 1-1 Hachioji, Tokyo 192-0397, Japan

\section{A. Balduino}

Development \& Technology Research Center, Universidade Veiga de Almeida, Rio de

Janeiro, RJ 20271-020, Brazil and AL initially modulate different metabolic and physiological programs, which exclusively over time generate two biologically different organisms. Such distinct diet-related senescence is analogous to the divergent aging processes and causes of death observed between castes of social insects, such as queens versus workers ("caste-related-senescence") and also between breeding versus non-breeding semelparous animals ("reproduction-related-senescence"). All these aging phenotypes are different not because they accumulate changes at a different rate, but because they accumulate different changes over time. Thus, the environment does not simply affect the individual aging rate through stochastic effects (e.g. U.V.) but also modulates the activation of a particular program/strategy that influences lifespan (e.g. caste, calorie intake). We refer to the environment-dependent aging patterns encoded by the genome as "senemorphism". Based on this idea we propose experimental schemes for aging, evolution and biomedical research.

Keywords Senemorphism - Caloric restriction · Longevity $\cdot$ Biomarkers $\cdot$ Senescence $\cdot$ Evolution

\section{Senemorphism (genetically molded aging patterns)}

While aging appears to be an almost universal feature of life, aging rates and lifespans show great diversity 
even within a given species. A hypothetical example of a straightforward delay of an aging process would be where the process is the result of simple chemical reactions: A temperature-dependent difference in lifespan within species, for example, can be explained in part by the chemistry of life: lower temperature decelerates chemical reactions, resulting in an extended lifespan due to the slower speed of the degenerative processes (slower aging rate). In this case, the changes responsible for aging are supposed to be the same in the long-lived and short-lived individuals, only the reaction rates change. In other cases there appear to be two or more independent aging patterns, each dependent on differences in gene expression promoted by extrinsic factors. Here we demonstrate that most, if not all, species have such an adaptive and parallel evolution of age-related phenotypes. We propose the new term "senemorphism" to refer to a plastic aging phenotype which can be generated through genetic pathways in response to environmental conditions. This is a novel and vital concept, and the objective of this study is solely to: (a) highlight convincing evidence that longevity plasticity encoded by the genotype as a general phenomenon exists, and (b) to make the case that diet-related changes in aging are an important example of the same phenomenon. Based on this idea we will also allude to experimental schemes for aging, evolution and biomedical research.

In the following two sections we show that senemorphism exists by considering well-accepted examples of how aging is affected (via genetic pathways) by the environment. Note that, in this study, we will not attempt to propose an explanation for how or why different patterns of aging evolved.

\section{The case for senemorphism: caste}

Senemorphism can be seen in social species, which are easily recognized by their adaptive and parallel evolution of age-related phenotypes. Different castes (soldiers, supersoldiers, workers, queens) evolved different lifespans, which most likely are correlated with their specific functions; as proposed by Hamilton (1966). Lasius niger sterile worker ants, for example, typically exhibit lifespans of 1-3 years whereas highly active reproducing queens with the same genetic background may live over 20 years (Keller and
Genoud 1997). We refer to this phenomenon as “caste-related senemorphism" (Fig. 1).

\section{The case for senemorphism: reproduction}

Reproduction-related senemorphism is most clearly seen in semelparous animals. Most semelparous mammals die during their first year, around 2 weeks after reproduction following an extremely rapid degenerative process. However, when reproduction is denied, the lifespan of these animals can increase several-fold and resembles a "normal" degenerative process (Schmidt et al. 2006; Woolley 1966). This we refer to as "reproduction-related senemorphism" (Fig. 2a). Functional disturbance of or removal of sexual organs in semelparous animals (as well as
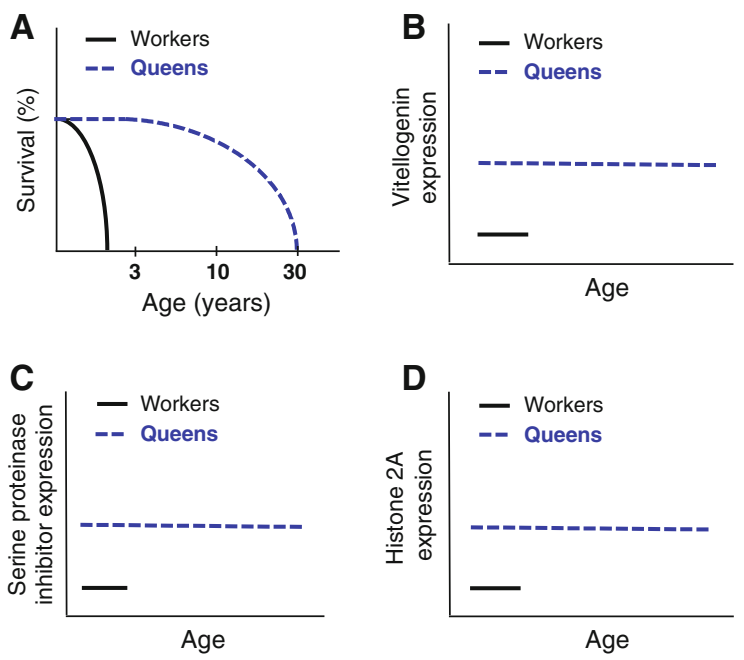

Fig. 1 Differential gene expression controls caste-related senemorphism in ants (Gräff et al. 2007). a-d Lifespans and the constitutive higher gene expression related to longevity in ant queens (blue) compared to workers (black). a The longevity of L. niger workers versus queens (Gräff et al. 2007). b Different gene expression levels of vitellogenin over the lifespans of queens and workers. Increased vitellogenin expression is thought to lead to improved body maintenance and thus contribute towards increased lifespans. (Seehuus et al. 2006). c Relative expression of serine proteinase inhibitor genes in workers and queens. Higher levels of serine proteinase inhibitor are thought to be immunoprotective (Kanost 1999). d Histone $2 \mathrm{~A}$ gene expression in queens versus workers. Higher histone $\mathrm{H} 2 \mathrm{~A}$ levels are thought to be responsible for improved maintenance of chromatin structure (Gräff et al. 2007). In addition, all of these genes are thought to have roles in strengthening the immune system 
A
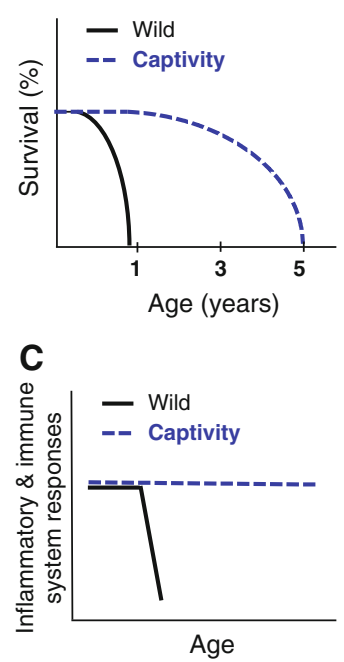

B

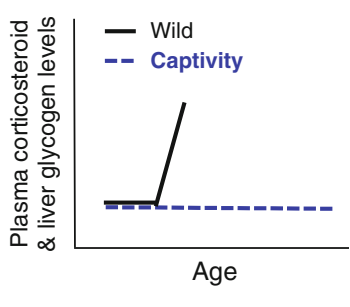

D

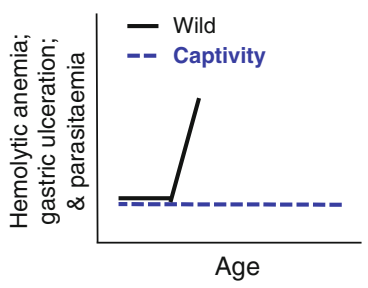

Fig. 2 Evidence of two different genetic programs controlled by the environment generating different senemorphs. Life history death strategy during or shortly after a single breeding season is the lifespan-limiting factor for wild (black) semelparous animals such as semelparous dasyurids (Schmidt et al. 2006). In fact, semelparous animals have the potential to exhibit a normal aging process in captivity (blue). a Wild semelparous dasyurids live $<1$ year, dying mainly during the breeding season by activation of a death program. Captured animals are able to live up to 5 years (Bradley 2003). b Abrupt endocrine and physiological changes can be observed during death program activation, for example increases of cortisol and liver glycogen (Barnett 1973). c In wild animals, abrupt physiological changes cause diverse pathologies for example shut down of immune system and inflammatory responses (Barker et al. 1978; Cheal et al. 1976). d Remarkable increases of gastric ulceration, haemolytic anaemia and parasitemia observed in wild animals prior to death (Barker et al. 1978; Bradley 1997)

removal of the seeds from semelparous, annual plants) prolongs their lives (Robertson 1961; Wareing and Seth 1967; Wodinsky 1977). Interestingly, the females of some semelparous species of octopus die within days of hatching of their young, but removal of the optic glands after reproduction has taken place results in the reestablishment of normal behavior and increased lifespan (Wodinsky 1977). These examples show that the trigger that kills a semelparous animal is controlled at least partially by environmental conditions with the animals themselves in fact retaining the ability to maintain their physical integrity for longer periods. In other words, these animals can die due to a clear "death strategy" or die exhibiting a slower aging process. The death strategy of semelparous animals is related to extreme hormonal changes, increase of inflammation and a downgrading of the immune

system (Humphries and Stevens 2001; Oakwood et al. 2001; Stein-Behrens and Sapolsky 1992; Wingfield and Sapolsky 2003). Non-breeding semelparous animals do not show these extreme changes because they undergo a different senemorphosis (Fig. 2b-d). In summary, there are several well-known species that show clear plasticity of lifespan due to changes in gene expression and hormonal levels, which appear to have evolved as an adaptation to a specific environment and result in at least two distinct routes to death.

\section{Diet-related senemorphism}

In this section we present the case that diet (energy intake) is an important environmental factor determining aging phenotypes as is illustrated by the extreme differences between caloric restriction (CR) and ad libitum (AL) organisms.

The discovery that animals undergoing CR live longer than those undergoing AL (McCay and Crowell 1934) (Fig. 3a) was one of the most remarkable in
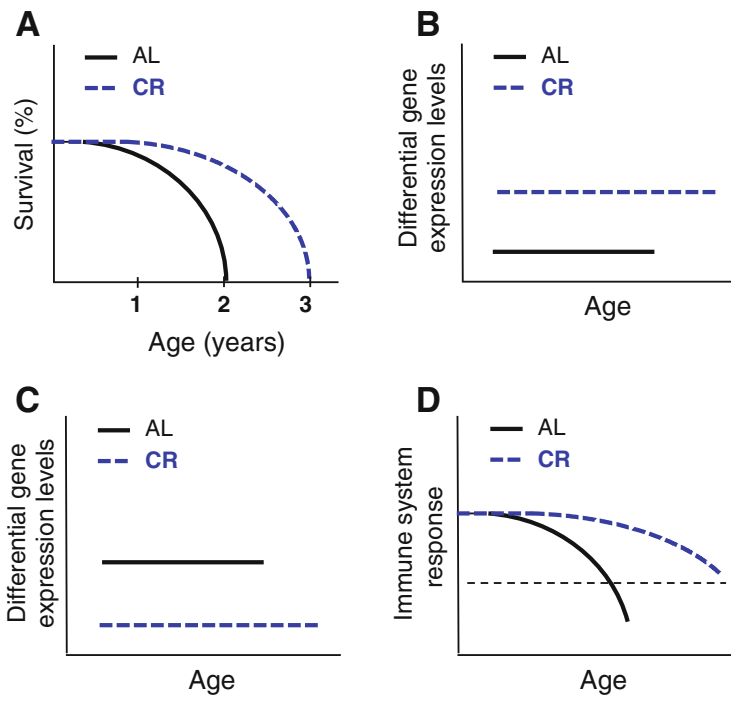

Fig. 3 Comparison of lifespans and gene expression throughout total lifespan in AL (black) and CR (blue) mice, from birth to death based on the work of Cao et al. (2001). a CR mice live $50 \%$ longer than AL mice. b Some genes (e.g. Ubp1, Got 1, Hal, Sultn) remain expressed at a higher levels throughout life in CR mice. c Some genes (e.g. hsp86-1, hspa5, ly6e, xbpl) remain expressed at a higher level throughout life in AL mice. d Immune senescence is delayed by CR. Importantly, at the time of death the immune system most likely remains more efficient in CR animals (dotted line) (Chen et al. 1998) 
aging research. The pathways involved in aging during $\mathrm{CR}$ or AL exhibit plasticity in response to extrinsic factors (Anderson and Weindruch 2007). They are ultimately controlled and coordinated by energy sensing mechanisms that are evolutionarily conserved from unicellular life forms to mammals (Flatt and Schmidt 2009; Fontana et al. 2010; Rose 2009; Rose and Nusbaum 1994). A commonly accepted hypothesis is that this response evolved to enable organisms enduring famine to survive until food returns and they can successfully reproduce (Holliday 1989). In contrast, under AL conditions reproduction is optimized at the expense of longevity (Kirkwood and Rose 1991; Holliday 1989; Masoro 2003; Merry and Holehan 1979). Because, generally, CR delays senescence compared with $\mathrm{AL}$, the conventional conclusion is that the aging under CR conditions is simply a delay of the aging under AL conditions (as may be the case for a slower aging rate under low temperature). The alternative is that, as in social and semelparous species, aging under AL and CR are distinct processes related to metabolic differences rather than metabolic rate (Helfand et al. 2008; Braeckman et al. 2006). This hypothesis has been addressed experimentally in the case of short-lived animals (Drosophila). Mair et al. (2003) elegantly demonstrated that temperature and diet-related aging processes are fundamentally distinct: Whereas low temperature is related to a simple slowing in the mortality rate, calorie intake is clearly a diet-dependent adaptation of a specific lifespan strategy. Diet is already well known to influence the morphogenesis of organisms, affecting for example the final adult size, color, caste and even sexual differentiation. Here we highlight the commonly overlooked fact that the influence of diet extends to lifespan and remains throughout maturation, possibly until death. We call this "diet-related senemorphism."

Diet-related senemorphism can be clearly identified through analysis of experimental data which shows clear and important differences between CR aging and $\mathrm{AL}$ aging. As in the case of the longer lifespan examples of caste and reproduction-related senemorphisms, CR animals show similar differences in gene expression, hormone levels and phenotypes (Cao et al. 2001; Higami et al. 2004) (Figs. 1, 2, 3). Several of these differences remain throughout the whole life of an organism or run in an opposite direction in CR compared to AL. CR animals can exhibit age-related changes and phenotypes that cannot be seen in $\mathrm{AL}$ animals even if diets are subsequently switched (Cao et al. 2001). These distinct diet-related aging processes are incompatible with the assertion that aging under $\mathrm{CR}$ is simply a delayed version of AL aging. Distinct aging processes are characterized by accumulation of distinct changes over time. By definition AL and CR aging are distinct processes and therefore examples of senemorphism. Evidence demonstrating diet-related senemorphism is discussed below.

\section{Evidence for diet-related senemorphism}

In this section we present evidence to support the idea that $\mathrm{CR}$ aging is a fundamentally different process than $\mathrm{AL}$ aging and that different caloric intakes generate different senemorphs (specific aging phenotypes).

Anderson and Weindruch (2007) elegantly described metabolic reprogramming in $\mathrm{CR}$, corroborating the idea that starvation generates different phenotypes. The fact that CR and AL phenotypes are completely different does not exclude the possibility that the age-related changes could occur in the same direction, but at a slower rate. However, if this was the case and CR aging was simply delayed AL aging, then we could easily predict that every age-related change seen during AL aging would occur with similar direction and magnitude and at proportionately the same time relative to total lifespan in CR aging. Most of these predictions could be imagined to hold for some species undergoing idealized temperature-related aging but do not apply for dietrelated aging. Instead, several changes over time are clearly diet-specific.

It is important to note that extension of lifespan is positively related to a decreasing calorie intake along a continuum down to a lower calorie limit. Thus under intermediate calorie intakes, intermediate senemorphs are to be expected. While this remains to be confirmed, here we simplify the discussion and consider, as is usually the case with most CR studies, solely the dietrelated adaptations at the two extremes: we consider AL aging as the "normal standard" for the fastest aging senemorph because it is adapted to optimize reproduction (analogous to the reproduction-triggered accelerated death in semelparous species); we consider optimized CR aging as the "normal standard" for the slowest aging senemorph because optimized CR aging maximizes lifespan. 
Examples accounting for these distinct senemorphoses include:

(1) Oxidative damage. The positive relationship between aging and the accumulation of oxidative damage is well known (Finkel and Holbrook 2000). However, CR is able to suppress completely the age-related accumulation of oxidative damage in some tissues (Lass et al. 1998) (Fig. 4a). More remarkably, switching old AL mice to a CR diet can reverse the age-related accumulation of oxidative damage in some tissues (Forster et al. 2000) (Fig. 4a). If CR was simply delayed AL aging, a slowed rate of accumulation of oxidative damage in all tissues rather than a maintenance or a reversal effect would be expected.

(2) Several genes that show age-related increases or decreases in expression during $\mathrm{AL}$ aging, show a different or even an antagonistic expression pattern during CR aging (Cao et al. 2001). In some cases the divergence between expression patterns increases as the period of CR lengthens (Fig. 4b). Interestingly, most of the genes that show a difference in expression are related to processes implicated in longevity (e.g. oxidative stress, inflammation, DNA repair, chaperones, metabolism, apoptosis, cell senescence, protein turn-over) (Cao et al. 2001; Coyle and Kroll 2008; Labinskyy et al. 2009; Swindell 2009). These age-related differences in gene expression profiles alone strongly support the existence of diet-related senemorphism.

(3) Stem cells lose their repopulation capacity during AL aging. Unexpectedly, short-term CR increases this potential; moreover long-term CR increases it further (Chen et al. 2003) (Fig. 4c).

(4) As most hormones are involved in growth, homeostasis and lifespan potential, it is not surprising that they are strongly affected by CR. The hormonal differences between animals undergoing $\mathrm{AL}$ and $\mathrm{CR}$ are well known. A number of studies have shown hormone levels that move in opposite directions over time in $\mathrm{AL}$ versus CR. For example, during AL aging, levels of hormones such as leptin increase whereas adiponectin and melatonin decrease, but possibly are driven in the opposite direction during long-term CR aging (Bedard et al. 2010; Chiba et al. 2002; Longo and
Fontana 2010; Roth et al. 2001) (Fig. 4d). Hormones such as insulin, IGF-1, DHEA, GH, ghrelin and cortisol are kept at healthy levels in CR animals (Heilbronn and Ravussin 2003) and shifts can occur in some of them when diet is switched from AL to CR (Dhahbi et al. 2004; Walford et al. 2002). Therefore most hormonal changes (or lack of changes) during senescence are specific to the period of diet and not purely due to chronological age or current diet status. Similarly, reproduction itself triggers the stress-related hormonal changes of accelerated deterioration that lead to semelparous death (Humphries and Stevens 2001; Oakwood et al. 2001; Stein-Behrens and Sapolsky 1992; Wingfield and Sapolsky 2003).

(5) The rate of fitness loss (deterioration in skin and hair condition, arthritis, cataracts and other sensory deterioration, muscle weakness, mobility, locomotion, cage climbing, sociality, etc.) in AL animals also follows a different pattern, varying in extent and relative time of onset when compared to CR animals. Therefore, there is no reason to expect to be able to predict the age of degeneration of a particular trait in CR through comparison of its degeneration under AL (e.g. although old AL mice show deterioration in muscle power, even very old CR mice do not). This uncertainty automatically invalidates the assumption that CR aging is a delay of AL aging. (Dubey et al. 1996; Fowler et al. 2002; Hepple et al. 2005; Someya et al. 2007) (Fig. 4e).

(6) Cause of death: As seen for breeding versus nonbreeding semelparous animals and workers versus queens of social insects, AL and CR animals die from different causes (Shimokawa et al. 1993) (Fig. 4f). This is to be expected only if the two processes are fundamentally different rather than differing only in the speed at which they occur. Where causes of death for CR animals are known, their frequency of occurrence does not follow the same pattern as in AL animals (Sell et al. 2000). Thus, CR is not a delay of AL; the genetic component of the CR program is fundamentally different, leading to a different aging process over time (senemorphism). The outcomes of these aging processes are two biologically different organisms (Fig. 4). In comparison to their respective younger ages, older $\mathrm{AL}$ and $\mathrm{CR}$ animals have undergone a clearly different senemorphosis, which 

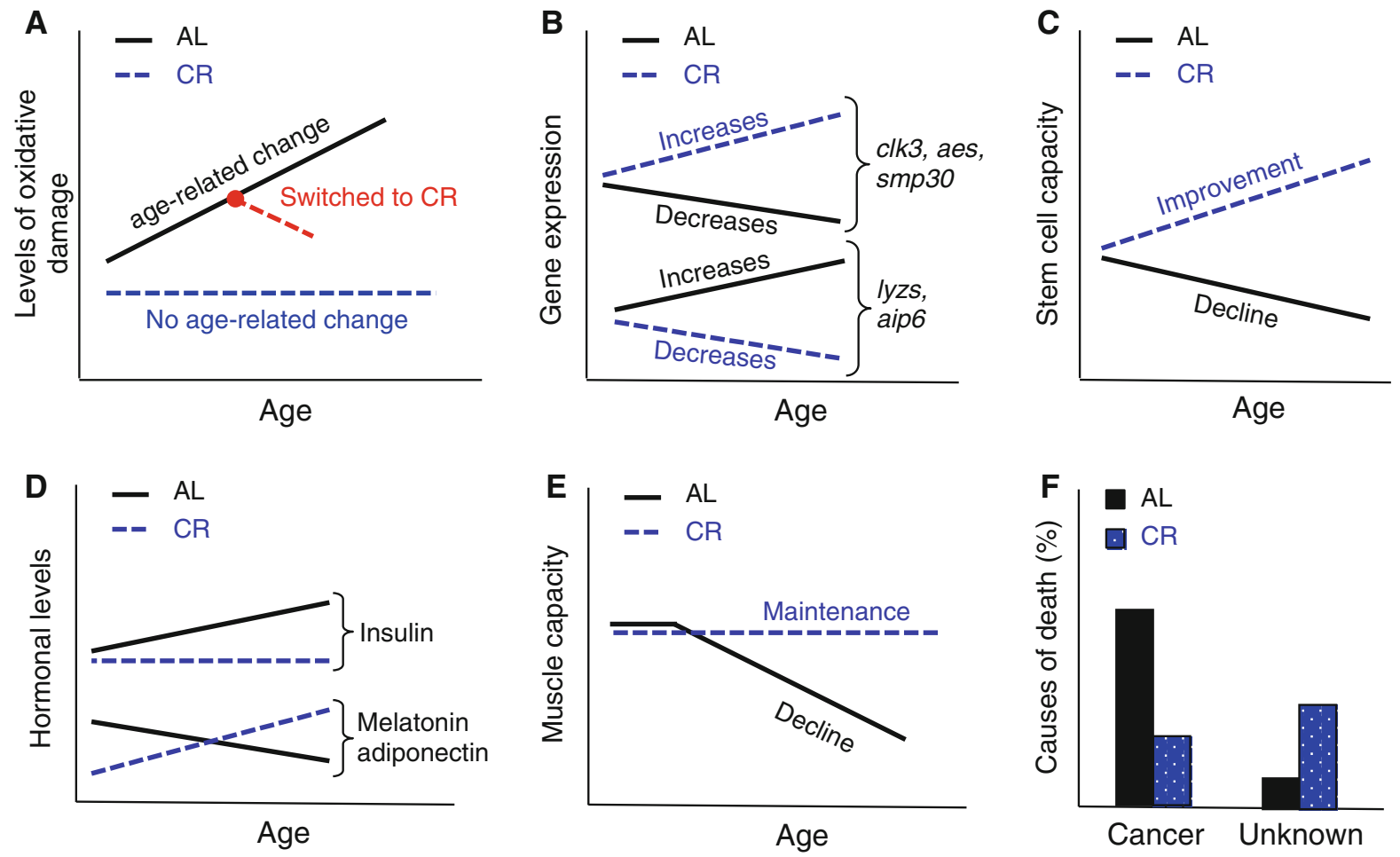

Fig. $4 \mathrm{CR}$ and $\mathrm{AL}$ are different aging processes which are characterized by distinct senemorphisms. a Accumulation of oxidative damage during $\mathrm{CR}$ and $\mathrm{AL}$ aging: in some cases no age-related change is observed during CR aging (Lass et al. 1998) (blue line). In other cases, switching old AL animals to $\mathrm{CR}$ (red line) may even reverse the accumulated damage (Forster et al. 2000). b Depending on tissue (in this case liver) some genes like $c l k 3$, aes and smp30 increase their expression during CR aging, but decrease their expression during AL aging. The opposite also can occur for example with lyzs and aip6 genes (Son et al. 2006). c The repopulation capacity of stem cells is well known to decrease during AL aging. Interestingly, this capacity improves during CR aging (Chen et al. 2003).

is only possible due to their longevity plasticity encoded by the genome. From the nature of the genes affected, CR, would be expected to have an effect analogous to that observed in queens of eusocial insects and captive semelparous animals, namely a life-prolonging effect, while AL would have an antagonistic effect akin to that seen in social insect workers and breeding semelparous animals, namely shorter lifespans (Figs. 1, 2, 3). This similarity may not be a coincidence: It may indicate that, as in caste-related and reproduction-related senemorphism both the longer and shorter lifespans of these diet-related senemorphic strategies are specific adaptations to the niche. Below we d Most hormones levels remain different in AL versus CR aging. For example insulin levels increases during AL aging, but show no age-related change during $\mathrm{CR}$ aging. Adiponectin seems to increase during $\mathrm{CR}$ aging, but decrease during $\mathrm{AL}$ aging and the same may be true for melatonin (Chiba et al. 2002; Dhahbi et al. 2004; Heilbronn and Ravussin 2003; Roth et al. 2001; Walford et al. 2002). e Fitness loss during CR aging is very different to fitness loss during AL aging. CR animals seem to keep their muscle function until the end of life, while AL animals gradually lose muscle function (Hepple et al. 2005). f AL and CR animals die for different reasons; most AL mice die due to cancer, while higher frequency of CR animals die due to unknown causes (Shimokawa et al. 1993)

highlight the importance of the concept of senemorphism for biological research.

\section{Implications: distinct senemorphisms—distinct approaches}

Here we consider the implications of diet-related senemorphism on perceptions of aging and research in the field.

Although CR research has progressed considerably in the past 100 years, the intuitive idea that $\mathrm{CR}$ is a delay of $\mathrm{AL}$ is still common. This has inhibited our ability to understand both aging processes. It is 
clear that the amount of calories consumed affects the gene expression profile, which distinctly modulates senemorphosis in most species (species that respond to CR). In other words, multiple senescence patterns modulated by the genome in response to food intake have evolved. This idea has a profound and important effect on how we think, study and analyze aging and age-related diseases. It is most likely the case that the longevity plasticity seen in senemorphic strategies is an adaptation to different environmental conditions; similar ideas have been described (Demetrius 2005; Carnes 2011). Thus, fully fed (AL) animals cannot simply be considered as a standard control for aging studies, an idea previously discussed by Le Bourg (2003). Note that, as elegantly described by Demetrius (2005), different species most likely had adapted divergent mechanisms to increase lifespan under CR conditions. Consequently, it is probably the case that different species also manifest distinct side effects of optimized reproduction under AL conditions. In agreement with Demetrius (2005), we support the idea that the evolution of senemorphic pathways in different species is most likely related to the species-specific life history. Therefore, if diet-related longevity is associated with species-specific metabolic pathways rather than the metabolic rate itself, comparisons between species should be carefully conducted.

Since the amount of food intake alters the metabolism, physiology and the aging process itself, generating different phenotypes, it is crucial for biological studies mainly associated with evolution, aging and age-related diseases to take food into account. As a minimum, the following should be considered: (1) quantity of food should always be measured; (2) a pair feed control group should always be conducted; (3) the same experiment should be carried out under at least the two extreme calorie intakes conditions. For example, the effectiveness of a drug or treatment could depend on high-level expression of SMP30 or stem cell potential. Thus the effectiveness would be enhanced in animals subjected to long-term CR as they show higher levels of SMP30 and stem cell potential compared to young individuals (Fig. $4 \mathrm{~b}, \mathrm{c}$ ). Conversely it may be ineffective for animals subject to long-term AL as their levels decrease below those of the young individuals (Fig. 4b, c). In summary, what is true for individuals/populations under long-term ("normal") AL may be completely false for individuals/populations under long-term CR.

Each diet-related senemorph (even if it is one "snapshot" from a continuum of phenotypes) has its own age-related biology and will most likely have its own fitness value from an evolutionary perspective. Therefore we suggest that all biological studies should take into account which diet-related senemorph is being studied. For instance, by analyzing ant workers we cannot expect to understand ant queens: each morph has a different biology and is adapted to different environments.

$\mathrm{CR}$ and $\mathrm{AL}$ aging generate different age-related phenotypes, therefore the way in which $\mathrm{CR}$ aging is investigated in comparison to AL becomes of vital importance. CR animals for example, should be studied until their natural death and not only for the duration of a "normal" (AL) lifespan. Data from old CR animals should always be compared with young CR animals and not with young AL "control" animals [interesting examples of experimental designs can be found in the literature (Cao et al. 2001; Fontana et al. 2010; Hepple et al. 2005; Lass et al. 1998)]. When AL and CR animals are compared, it would be preferable to compare animals at the same developmental stage rather than the same chronological age (as is done when carrying out inter-species comparisons; Fig. 5). If we compare animals with the same chronological age, the life-prolonging effects of CR will be confounded with the changes related to delayed growth and senescence. For example, comparing a CR animal at 7.4 months old (just matured) with an AL animal at 1.3 months old (just matured) would easily distinguish between the changes related to delayed maturation and the changes related to the life-prolonging affects of $\mathrm{CR}$; such as, increases of stem cell potential or SMP30 (avoiding wrong conclusions) (Fig. 4). The developmental stage itself may dictate the optimal level of CR; there is no reason to feed the $\mathrm{CR}$ group according to the AL chronological requirements, if the duration of growth, maturation and reproductive senescence (RS) varies asymmetrically between the two groups (Fig. 5). The calorie intake being dynamically related to AL developmental stage rather than AL chronological age may determine the real maximal lifespan and has not been investigated to date. This approach also would allow facile identification of age-related changes commonly shared by $\mathrm{CR}$ and AL animals and, importantly, distinguish the life-prolonging 


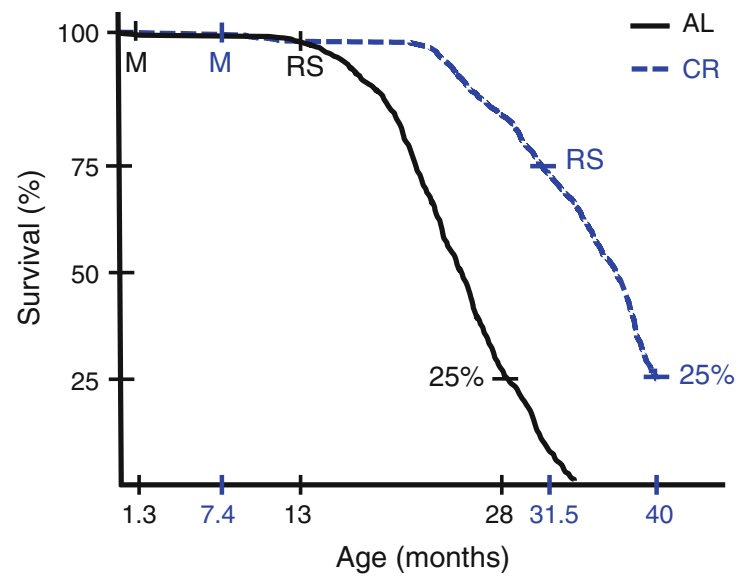

Fig. 5 Timing of developmental stages differs in AL and CR rats (adapted from Merry and Holehan 1979). CR does not merely delay the aging process itself: Compared to the $\mathrm{AL}$ group, CR lengthened the maturation $(M)$ period by $470 \%$, lengthened the period from maturation to RS by $100 \%$ and reduced the period from RS to $25 \%$ survival by $50 \%$. Although these animals are from the same species, CR has apparently acted on energy sensing mechanisms to modify several important genetic pathways related to reproduction and longevity. These AL and CR animals differ more physiologically and metabolically than closely related species which are aging while sharing a common diet. Thus comparison of animals with the same chronological age but on different diets may not be the correct comparison to make. This is because the changes related with life-prolonging effects of $\mathrm{CR}$ will be masked among the changes related to delayed growth and senescence

effects of those changes specifically related to the rate of aging from those related to senemorphism.

Because CR aging causes a cascade of specific agerelated changes over time it would be reasonable to search for specific diet-related biomarkers of aging (Fig. 4). For instance, age-related decreases in stem cell potential within the AL group seems be an anticancer strategy. Consequently, it could be the case that age-related decreases of stem cell potential within the AL group are correlated with longer lifespan. CR animals increase the expression of anti-cancer genes, allowing increases of stem cell potential to enhance regeneration without promoting cancer. In this case, in contrast to the AL group, increases of stem cell potential within the $\mathrm{CR}$ group could be correlated with better regeneration and longer lifespans. We strongly support the idea that biomedical research will continually have difficulties to find a reasonable aging biomarker for humans while data from individuals with completely different calorie intakes are not segregated.
It is likely that CR will provide similar benefits to humans as seen in other mammals, however, following a CR regimen is difficult in areas where food is plentiful. Therefore a CR mimetic drug is an attractive option. Putting aside the fact that the changes seen in CR are multifaceted and may not be easy to emulate with a single therapeutic, diet-related senemorphisms raise another serious hurdle: Because $\mathrm{AL}$ and $\mathrm{CR}$ aging are different processes with widely differing biochemistries, a drug which mimics one part of the CR pathway but in an AL background may have unpredictable effects. For example: the age-related increase of stem cell potential in CR animals could be responsible for the optimized regeneration in these animals. Would it be sensible to mimic increases of stem cell potential in $\mathrm{AL}$ animals? Possibly not; CR increases stem cell potential but also increases protection against cancer. Under AL, however, protection against cancer decreases. A CR mimetic drug that increases stem cell potential in AL animals could quite possibly be cancer-inducing.

\section{Final considerations}

We cannot regard aging as a single process, rather the aging phenotype depends on how environmental conditions influence gene expression that will modulate a specific senemorphosis. This has important experimental implications. For example in aging mice we can ask questions such as: do most aging mice develop cancer? Do stem cells lose their efficiency? Does the level of senescent marker protein 30 decrease? Do muscles weaken? The answers are "yes" in all cases for the fully fed (AL) mice phenotype and "no" in all cases for the optimized CR mice phenotype (Fig. 4). These distinct senemorphs are not related to the current dietary status, but to the long-term accumulation of changes under different dietary conditions. Noticeably, most agerelated changes in the $\mathrm{CR}$ phenotype remain disproportionate to or run in an opposite direction to changes seen in the AL phenotype (Fig. 4). Much more experimental work needs to be carried out to further understand the differences between the two longevity strategies.

Previously published experiments on aging and evolution may also benefit from reanalysis in the light 
of life-history senemorphism. We expect that an awareness of the existence of diet-related senemorphic processes will accelerate biomedical research and specifically help us to understand the causes of natural death and death by disease. We believe the characterization of distinct age-related biology (senemorphism) as an adaptation to specific diets creates a new perspective on philosophy, evolution and biology.

Acknowledgments We thank Emilio Jeckel Neto and Italo Oliveira Santos for the inspiration to address this problem. We thank Isao Shimokawa for the decisive initial discussion, incentive and supporting the development of this manuscript. We thank Taro Kaneuchi, Aubrey De Grey, Ana Maria Suzuki and Ivana Mânica da Cruz for constructive discussions. We thank Isao Shimokawa, Alexandre Peixoto and Junjiro Horiuchi for critical reading of the manuscript.

Open Access This article is distributed under the terms of the Creative Commons Attribution License which permits any use, distribution, and reproduction in any medium, provided the original author(s) and the source are credited.

\section{References}

Anderson RM, Weindruch R (2007) Metabolic reprogramming in dietary restriction. Interdiscip Top Gerontol 35:18-38

Barker IK, Beveridge I, Bradley AJ, Lee AK (1978) Observations on spontaneous stress-related mortality among males of the dasyurid marsupial antechinus stuartii macleay. Aust J Zool 26(3):435-447

Barnett JL (1973) A stress response in Antechius stuartii (Macleay). Aust J Zool 21:501-513

Bedard K, Robinette K, Ferland G, Gaudreau P (2010) Effects of long-term dietary interventions on pituitary growth hormone-releasing hormone receptor in aging rats and potential mechanisms of action. Mech Ageing Dev 131(3): $169-178$

Bradley AJ (1997) Reproduction and life history in the red-tailed phascogale, Phascogale calura (Marsupialia: Dasyuridae): the adaptive-stress senescence hypothesis. J Zool 241(4): 739-755

Bradley AJ (2003) Stress, hormones and mortality in small carnivorous marsupials. In: Jones M, Dickman C, Archer M (eds) Predators with pouches - the biology of carnivorous marsupials. CSIRO Publishing, Victoria, pp 255-267

Braeckman BP, Demetrius L, Vanfleteren JR (2006) The dietary restriction effect in C. elegans and humans: is the worm a one-millimeter human? Biogerontology 7:127-133

Cao SX, Dhahbi JM, Mote PL, Spindler SR (2001) Genomic profiling of short- and long-term caloric restriction effects in the liver of aging mice. Proc Natl Acad Sci USA 98(19): 10630-10635

Carnes BA (2011) What is lifespan regulation and why does it exist? Biogerontology 12(4):367-374
Cheal PD, Lee AK, Barnett JL (1976) Changes in the haematology of antechinus stuartii (Marsupialia), and their association with male mortality. Aust J Zool 24(3):299-311

Chen J, Astle CM, Harrison DE (1998) Delayed immune aging in diet-restricted B6CBAT6 Fl mice is associated with preservation of naive T cells. J Gerontol A 53A(5):B330B337

Chen J, Astle CM, Harrison DE (2003) Hematopoietic senescence is postponed and hematopoietic stem cell function is enhanced by dietary restriction. Exp Hematol 31(11): $1097-1103$

Chiba T, Yamaza H, Higami Y, Shimokawa I (2002) Anti-aging effects of caloric restriction: involvement of neuroendocrine adaptation by peripheral signaling. Microsc Res Tech 59(4):317-324

Coyle Scott, Kroll Evgueny (2008) Starvation induces genomic rearrangements and starvation-resilient phenotypes in yeast. Mol Biol Evol 25:310-318

Demetrius L (2005) Of mice and men. EMBO Rep 6:S39-S44

Dhahbi JM, Kim HJ, Mote PL, Beaver RJ, Spindler SR (2004) Temporal linkage between the phenotypic and genomic responses to caloric restriction. Proc Natl Acad Sci USA 101(15):5524-5529

Dubey A, Forster MJ, Lal H, Sohal RS (1996) Effect of age and caloric intake on protein oxidation in different brain regions and on behavioral functions of the mouse. Arch Biochem Biophys 333(1):189-197

Finkel T, Holbrook N (2000) Oxidants, oxidative stress and the biology of ageing. Nature 408(6809):239-247

Flatt T, Schmidt PS (2009) Integrating evolutionary and molecular genetics of aging. BBA Gen Subj 1790(10): 951-962

Fontana L, Klein S, Holloszy J (2010) Effects of long-term calorie restriction and endurance exercise on glucose tolerance, insulin action, and adipokine production. AGE 32(1):97-108

Forster MJ, Sohal BH, Sohal RS (2000) Reversible effects of long-term caloric restriction on protein oxidative damage. J Gerontol A 55(11):B522-B529

Fowler CG, Torre P, Kemnitz JW (2002) Effects of caloric restriction and aging on the auditory function of rhesus monkeys (Macaca mulatta): the University of Wisconsin Study. Hear Res 169(1-2):24-35

Gräff J, Jemielity S, Parker JD, Parker KM, Keller L (2007) Differential gene expression between adult queens and workers in the ant Lasius niger. Mol Ecol 16(3):675-683

Hamilton WD (1966) The moulding of senescence by natural selection. J Theor Biol 12(1):12-45

Heilbronn LK, Ravussin E (2003) Calorie restriction and aging: review of the literature and implications for studies in humans. Am J Clin Nutr 78(3):361-369

Helfand SL, Bauer JH, Wood JG (2008) Calorie restriction in lower organisms. In: Guarente L, Partridge L, Wallace DC (eds) Molecular biology of aging. Cold Spring Harbor Laboratory Press, New York, pp 73-93

Hepple RT, Baker DJ, Kaczor JJ, Krause DJ (2005) Long-term caloric restriction abrogates the age-related decline in skeletal muscle aerobic function. FASEB J 19(10):1320-1322

Higami Y, Pugh TD, Page GP, Allison DB, Prolla TA, Weindruch R (2004) Adipose tissue energy metabolism: 
altered gene expression profile of mice subjected to longterm caloric restriction. FASEB J 18(2):415-417

Holliday R (1989) Food, reproduction and longevity: is the extended lifespan of calorie-restricted animals an evolutionary adaptation? BioEssays 10(4):125-127

Humphries S, Stevens DJ (2001) Reproductive biology: out with a bang. Nature 410(6830):758-759

Kanost MR (1999) Serine proteinase inhibitors in arthropod immunity. Dev Comp Immunol 23(4-5):291-301

Keller L, Genoud M (1997) Letters to nature. Extraordinary lifespans in ants: a test of evolutionary theories of ageing. Nature 389:3-5

Kirkwood TB, Rose MR (1991) Evolution of senescence: late survival sacrificed for reproduction. Philos Trans R Soc Lond B 332(1262):15-24

Labinskyy N, Mukhopadhyay P, Toth J, Szalai G, Veres M et al (2009) Longevity is associated with increased vascular resistance to high glucose-induced oxidative stress and inflammatory gene expression in Peromyscus leucopus. Am J Physiol 296(4):H946-H956

Lass A, Sohal BH, Weindruch R, Forster MJ, Sohal RS (1998) Caloric restriction prevents age-associated accrual of oxidative damage to mouse skeletal muscle mitochondria. Free Radic Biol Med 25(9):1089-1097

Le Bourg E (2003) Delaying aging: could the study of hormesis be more helpful than that of the genetic pathway used to survive starvation? Biogerontology 4(5):319-324

Longo VD, Fontana L (2010) Calorie restriction and cancer prevention: metabolic and molecular mechanisms. Trends Pharmacol Sci 31(2):89-98

Mair W, Goymer P, Pletcher SD, Partridge L (2003) Demography of dietary restriction and death in Drosophila. Science 301(5640): 1731-1733

Masoro EJ (2003) Subfield history: caloric restriction, slowing aging, and extending life. Sci Aging Knowl Environ 2003(8): re2

McCay CM, Crowell MF (1934) Prolonging the life span. Sci Mon 39:405-414

Merry BJ, Holehan AM (1979) Onset of puberty and duration of fertility in rats fed a restricted diet. J Reprod Fertil 57(2): 253-259

Oakwood M, Bradley AJ, Cockburn A (2001) Semelparity in a large marsupial. Proc R Soc B 268(1465):407-411

Robertson OH (1961) Prolongation of the life span of kokanee salmon (Oncorhynchus nerka kennerlyi) by castration before beginning of gonad development. Proc Natl Acad Sci USA 47(4):609-621
Rose MR (2009) Adaptation, aging, and genomic information. Aging 1:444-450

Rose MR, Nusbaum TJ (1994) Prospects for postponing human aging. FASEB J 8(12):925-928

Roth GS, Ingram DK, Lane MA (2001) Caloric restriction in primates and relevance to humans. Ann N Y Acad Sci 928(1):305-315

Schmidt AL, Taggart DA, Holz P, Temple-Smith PD, Bradley AJ (2006) Plasma steroids and steroid-binding capacity in male semelparous dasyurid marsupials (Phascogale tapoatafa) that survive beyond the breeding season in captivity. Gen Comp Endocrinol 149(3):236-243

Seehuus SC, Norberg K, Gimsa U, Krekling T, Amdam GV (2006) Reproductive protein protects functionally sterile honey bee workers from oxidative stress. Proc Natl Acad Sci USA 103(4):962-967

Sell DR, Kleinman NR, Monnier VM (2000) Longitudinal determination of skin collagen glycation and glycoxidation rates predicts early death in C57BL/6NNIA mice. FASEB J 14(1):145-156

Shimokawa I, Higami Y, Hubbard GB, McMahan CA, Masoro EJ, Yu BP (1993) Diet and the suitability of the male Fischer 344 rat as a model for aging research. J Gerontol A 48(1):B27-B32

Someya S, Yamasoba T, Weindruch R, Prolla TA, Tanokura M (2007) Caloric restriction suppresses apoptotic cell death in the mammalian cochlea and leads to prevention of presbycusis. Neurobiol Aging 28(10):1613-1622

Son TG, Zou Y, Jung KJ, Yu BP, Ishigami A, Maruyama N, Lee J (2006) SMP30 deficiency causes increased oxidative stress in brain. Mech Ageing Dev 127(5):451-457

Stein-Behrens BA, Sapolsky RM (1992) Stress, glucocorticoids, and aging. Aging Clin Exp Res 4:197-210

Swindell WR (2009) Genes and gene expression modules associated with caloric restriction and aging in the laboratory mouse. BMC genomics 10:585

Walford RL, Mock D, Verdery R, MacCallum T (2002) Calorie restriction in biosphere 2. J Gerontol A 57(6):B211-B224

Wareing PF, Seth AK (1967) Aging and senescence in the whole plant. Symp Soc Exp Biol 21:543-558

Wingfield JC, Sapolsky RM (2003) Reproduction and resistance to stress: when and how. J Neuroendocrinol 15(8):711-724

Wodinsky J (1977) Hormonal inhibition of feeding and death in octopus: control by optic gland secretion. Science 198(4320): 948-951

Woolley P (1966) Reproduction in Antechinus spp. and other Dasyurid marsupials. Symp Zool Soc Lond 15:281-294 